\title{
Application of Electrical Resistance Tomography
}

\author{
Chenhui Su ${ }^{1, a}$, Jianyu Zhao ${ }^{1, b}$, Hongwei Ren ${ }^{1, \star}$, Lei Qin ${ }^{2, c}$, Kaige Tian ${ }^{2, d}$ \\ ${ }^{1}$ Schools of Electrical Engineering, University of Jinan, Jinan 250022, P. R. China \\ ${ }^{2}$ School of Civil Engineering and Architecture, University of Jinan, Jinan 250022, P. R. China \\ asuchenhui2010@163.com, ${ }^{\mathrm{b}}$ cse_zjy@ujn.edu.cn, ${ }^{\mathrm{c}} \mathrm{cea}$ _qinl@ujn.edu.cn, ${ }^{\mathrm{d}} 327042822 @ q q . c o m$ \\ *cse_renhw@ujn.edu.cn
}

\section{Keywords: ERT; Image Reconstruction; Multiphase Flow; Medicine; Concrete}

Abstract. Electrical tomography technology is a kind of process tomography technology. It is accompanied by the development of computer technology and sensor technology. Electrical resistance tomography is a kind of mode of electrical tomography. It has some excellent characteristics that are non-invasive, portable, low cost and fast response etc. In this paper, the system of ERT unit are introduced. Mainly summarized the application of ERT system in the measurement of multiphase flow, medicine, the concrete structure detection and other aspects.

\section{Introduction}

Electrical tomography technology is one kind of PT technology which includes the electrical capacitance tomography, electrical resistance tomography, electrical impedance tomography and electromagnetic tomography [1]. As shown in Figure 1 principle of the ET system.

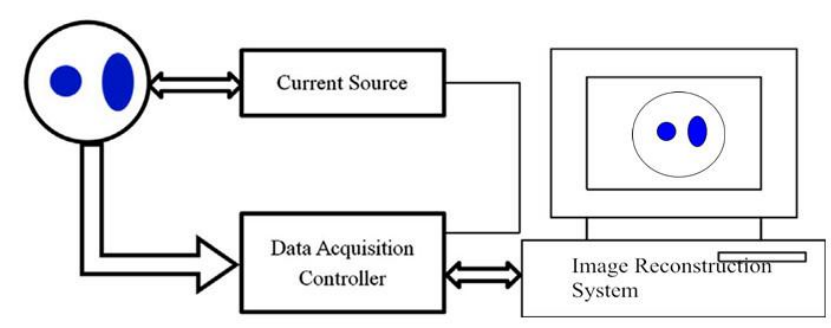

Fig.1. Principle of the ET system.

In general, the ET system consists of three parts: sensor (usually for space sensing electrode array), data acquisition system and image reconstruction. To obtain the information that are resistance, capacitance, resistance, permeability of the measured object is composed of a sensor. Then the signals are processed by the data acquisition system, and the signal is converted to digital quantity which is sent to the computer. It according to the image reconstruction algorithm, reconstruct the image of the object to be measured. For further analysis of reconstruction image, extracting the feature parameters and accomplishing the required parameters measurement.

After 20 years of development, has made much progress in three parts of electrical tomography system. The sensor, due to the requirements of different industrial processes are not the same, different sensor is designed, including: the sensor material selection, structural parameters of the sensor optimization design, the sensor structure and spatial layout, high temperature resistant, high pressure, abrasion resistance and corrosion resistance design etc. Domestic and foreign researchers have made great progress in the design of the data acquisition system. This research mainly experienced from analog to digital, serial to parallel acquisition, serial port to the USB transmission, single mode to collect multimodal image acquisition. A great progress has made in the image reconstruction algorithm. In view of the underdetermined problem, the soft field characteristic and the solution to the stability problem of the solution of the inverse problem, many feasible algorithms were studied. 
Electrical resistance tomography is different from the traditional CT technology [2]. It takes advantage of voltage or current on the field of imaging required incentive, then according to the external measurement of current or voltage value, within the field on the conductivity or resistivity inversion, so as to realize the imaging of the field distribution inside information. In fact, it is a simplified form of electrical impedance tomography, using only the real part of the impedance information. At the same time, need to point out that it is usually sense of electrical impedance tomography refers to electrical resistance tomography. Because at present both in the flow tomography and in medical engineering electrical impedance tomography mostly just using the real part of electrical impedance information, using the imaginary part information rarely, which is essentially electrical resistance tomography. This kind of imaging technology has low cost, easy to carry, fast imaging, non-intrusive, non-destructive measurement, ability of function imaging, and many other advantages, but in the design of the hardware, to overcome the "soft field" property and the development of reconstruction algorithm to research is needed.

At present, in the field of industrial real-time monitoring of pipeline in the multiphase flow in the field of medicine, functional imaging of human tissues as well as in concrete building structure monitoring, ERT has a great application and Development Research.

\section{The Theoretical Basis of Electrical Resistance Tomography}

The mathematical theory of electrical tomography technology is Radon transform and Radon inverse transformation for theoretical basis, the physical basis is based on the steady state electromagnetic field theory [3]. His mathematical model that the relationship between the distribution and boundary measurement signal measured the electric characteristics of the object derived from Maxwell's equations of electromagnetic field. The differential form:

$$
\left\{\begin{array}{l}
\nabla \cdot D=\rho \\
\nabla \cdot E=-\frac{\partial B}{\partial t} \\
\nabla \cdot B=0 \\
\nabla \cdot H=J+\frac{\partial D}{\partial t}
\end{array}\right.
$$

In the isotropic medium, there is

$\left\{\begin{array}{l}D=\varepsilon E \\ B=\mu H \\ J=\sigma E\end{array}\right.$

Where $\mathrm{D}$ is the electric induction, $\rho$ is the charge density, $\mathrm{E}$ is the electric field intensity, $\mathrm{B}$ is the magnetic induction intensity, $\mathrm{H}$ is the magnetic field strength, $\mathrm{J}$ is the current density, $\sigma$ is the conductivity, $\varepsilon$ is the dielectric constant, ${ }^{\mu}$ is the magnetic permeability.

There is a forward problem and inverse problem of electromagnetic field analysis in ET system. For some physical processes and phenomena, if the distribution of known physical process, according to the specific conditions where is the initial or boundary conditions of the state variables of the system to determine the variation of the system state variables, called the forward problem. The process of the opposite is called inverse problem.

For electrical resistance tomography system, it is already known and the sensitive field distribution of initial field and boundary conditions, calculate the distribution of electromagnetic field. Based on the theory of electromagnetic field, and in order to make the problem tractable, can make the following assumption of ERT problem research:

1. Sensitive field of ERT system as a quasi stable field. That is to say, when applying sinusoidal ac excitation around the field that current caused changes in the electric field around at the same time. That is to ignore the current from one point to another point of time. 
2. Because of the sensitive field of no excitation current sources or sinks current frequency range, thus around the sensitivity field the current divergence is 0 . Because of the sensitive field of ERT internal no excitation source, no eddy current effect. This condition can be satisfied.

So, ERT sensitive field of an arbitrary point satisfies the formula (3) and formula (2-4):

$$
\begin{aligned}
& J=\sigma E \\
& \nabla \cdot J=0
\end{aligned}
$$

At the same time the formula (5) was established:

$E=-\nabla \phi$

Where $\phi$ is the potential distribution. $\phi$ satisfies the formula (6) and formula (7)

$$
\begin{aligned}
& \nabla \cdot(\sigma \cdot \nabla \phi)=0 \\
& \sigma \cdot \nabla^{2} \phi+\nabla \sigma \cdot \nabla \phi=0
\end{aligned}
$$

In uniform, linear, isotropic conductive medium, is a constant. So the equation can be simplified as the formula (8) Laplace equation.

$$
\nabla^{2} \phi=0
$$

ERT system is the forward problem of sensitive field of ERT can be described as formula (9):

$$
\left\{\begin{array}{l}
\nabla \cdot(\rho \cdot \nabla \phi)=0, \text { In the sensitive field where is } \Omega \\
\left.\rho \cdot \frac{\partial \phi}{\partial n}\right|_{\partial \Omega}=\mathrm{j} \text {,In the current injecting electrodes } \\
\left.\rho \cdot \frac{\partial \phi}{\partial n}\right|_{\partial \Omega}=-\mathrm{j} \text {,In the current extraction electrodes }
\end{array}\right.
$$

Where $\nabla$ is the gradient operator, $\Omega$ is the sensitive field, ${ }^{\partial} \Omega$ is the sensitive field boundary, ${ }^{j}$ is the boundary current density, ${ }^{\partial \phi / \partial n}$ is the derivative along the direction normal to the boundary.

Electrical resistance tomography of the inverse problem is that according to the distribution of electromagnetic field and boundary measuring signals (also called projection data) to calculate the field distribution characteristic parameters of electrical, and expressed in the form of image, also known as image reconstruction. ERT inverse problem is equivalent to the solution of integral equation, the solution of the integral equation for the nonlinear problem, so the electrical resistance tomography of inverse problem for nonlinear problem. The solution of the inverse problem has great difficulty in solving the indeterminacy, the soft field characteristic, and the stability of the solution.

The image reconstruction algorithm of ERT can have many classification methods. Based on the use of measuring data is divided into dynamic and static algorithms. This paper mainly introduces the following three methods.

1) the Landweber iterative algorithm[4].

The Landweber iterative algorithm is a variant of the steepest descent method. It has been widely used in optimization problem. Landweber iteration algorithm's goal is to minimize the following objective functional.

$$
f(\mathbf{G})=\frac{1}{2}\|\mathbf{S G}-\mathbf{V}\|^{2}=\frac{1}{2}(\mathbf{S G}-\mathbf{V})^{T}(\mathbf{S G}-\mathbf{V})
$$

The gradient is

$$
\nabla f(\mathbf{G})=\mathbf{S}^{T}(\mathbf{S G}-\mathbf{V})
$$

The steepest descent method to choose the negative gradient direction for iteration direction. Thus available Landweber iteration algorithm for iterative formula.

$$
\left\{\begin{array}{l}
\mathbf{G}_{k+1}=\mathbf{G}_{k}+\alpha \mathbf{S}^{T}\left(\mathbf{V}-\mathbf{S G}_{k}\right) \\
\mathbf{G}_{0}=\mathbf{S}^{T} \mathbf{V}
\end{array}\right.
$$


Where $G_{k}$ is the image gray value of the k iteration, is the initial value for the image gray, $G_{0}$ is the iteration factor.

2)sensitivity coefficient method.

In 1985, Japan's Fuji University of Murai and Kagawa to establish the finite element model based on sensitivity theory [5], and puts forward the sensitivity coefficient algorithm. The sensitivity coefficient matrix is calculated by equation (13):

$$
S_{m, n, x, y}=\int \frac{\nabla \phi_{m}}{I_{m}} \cdot \frac{\nabla \phi_{n}}{I_{n}} d x d y
$$

Where Sm,n, $\mathrm{x}, \mathrm{y}$ is when the $\mathrm{m}$ electrode for the excitation electrode, $\mathrm{n}$ electrode for measuring electrode pair, the coordinate of the corresponding sensitivity coefficient of $(x, y) \cdot{ }^{\nabla \phi_{m}}$ and ${ }^{\nabla \phi_{n}}$ are respectively M and $\mathrm{N}$ electrodes on the electrode as the incentive of electrodes, and the excitation current sensitive field potential distribution of Im and In.

The basic idea is to assume that the conductivity distribution change is small, think field approximation sensitive field distribution is the same. After calculated the sensitivity coefficient of the reference image matrix, on the original equation that is $\mathbf{V}=\mathbf{S G}$ directly by using the sensitivity coefficient matrix transpose for image reconstruction. That is:

$$
\mathbf{G}=\mathbf{S}^{T} \mathbf{V}
$$

Where $\mathrm{G}$ is the image gray level matrix, $\mathrm{V}$ is the voltage measurement, $\mathrm{S}$ is matrix of sensitive coefficients.

3)the Tikhonov regularization algorithm[6].

Tikhonov regularization algorithm is a widely used effective method for solving ill-posed problems, has received many successful applications. As a matter of fact the algorithm for solving the following target functional:

$$
\min J(\mathbf{G})=\|\mathbf{S G}-\mathbf{V}\|^{2}+\mu\|\mathbf{G}-\overline{\mathbf{G}}\|^{2}
$$

In the formula, $\mathrm{G}, \mathrm{V}$ and $\mathrm{S}$ are defined as formula (14). $\overline{\mathbf{G}}$ is the initial estimates for image gray scale, general to $0 . \mu$ is the regularization parameter. The purpose of the minimum deviation at the same time the original ill-posed inverse problem into a well posed minimization problem solving.

To solve the equation (15), available standard Tikhonov regularization algorithm solution.

$$
\mathbf{G}=\left(\mathbf{S}^{T} \mathbf{S}+\mu \mathbf{I}\right)^{-1} \mathbf{S V}
$$

\section{Application of Electrical Resistance Tomography}

Reported by domestic and foreign literature, the ERT technology has obtained the preliminary application in many industrial processes. Its application areas involved in multiphase flow monitoring and parameter measurement, clinical application and for concrete structure health monitoring, etc. ERT is out of the lab and to industrial field application development and gradually shows its superiority.

\section{Condition Monitoring and Its Parameters of Multiphase Flow Measurement.}

In petroleum, chemical, electric power, metallurgy, building materials, food and other industries, there are a large number of heterogeneous material mixed flow. Study the flow of the two (more) phase flow characteristics and change rules, and the related parameters for testing, for the relevant scientific research, design and the safe and efficient operation of the production process is of great significance.

Industrial production in the common system of two-phase flow which are gas-solid two phase flow, gas-liquid two phase flow, liquid-solid two phase flow, liquid-liquid two phase flow. Compared with the single phase flow, the flow characteristics of two phase flow is more complex, mainly reflects in: flow type is complicated, the interaction and relative speed between the phase interface, physical property change is bigger, the mathematical description is difficult, and so on. Cause is used to describe the flow of the multiphase flow parameters than the single-phase flow, main parameters are flow pattern, split phase holdup, speed and slip ratio, pressure and pressure drop and flow rate, density and so on. 
ERT technique was applied to the operation condition of the hydro-cyclone separator monitoring by the University of Leeds scholars. Using ERT system provided by the separator is a discrete phase distribution on cross section image of time to change course. In order to verify the mechanism of the hydro-cyclone separator model and optimization of parameter design provides a new basis. They use ITS company developed ITS 1000 type of ERT system prototype, installed in the British ceramic clay refining factory hydro-cyclone separator, the test was carried out [7]. ERT can be normal operation in the industrial environment, results confirmed that the ERT running status of the hydro-cyclone separator feasibility of online measurement and control. Of re-searchers at the University of Leeds in the ERT technique has been in a leading position, such as Dai Y et al, the first research ERT double cross section technique. And the three dimensional detection of gas liquid two phase flow experiment and succeed, confirmed the ERT double cross section system is practical, and analyzes the velocity field and image reconstruction of gas phase holdup[8]. In recent years, the United States also began a lot of research on electrical imaging technology, Mi W et al., design a set of voltage and current measurements of ERT system, able to solve the ERT technology is used for fluid when the electric conductor.

At home, in 2001, Tianjin university's research team, led by professor huaxiang wang, developed the TERT - 1, 2, 3, 4 type system prototype, under the system of gas liquid two phase flow and oil/water two phase flow in a lot of kinds of experimental research, concluded that ERT acquisition system can realize two phase flow detection and the specific flow pattern identification of two phase flow [9]. In 2007, tianjin university zi qiang cui and others designed a kind of excitation signal source based on the FPGA implementation without touching the hardware circuit, can realize the signal frequency, amplitude, phase Angle, change type, can meet the demand of different data acquisition mode of ERT system. Huaxiang wang, in 2009, designed a set of ERT/ECT based technologies such as detection of oil/gas two-phase flow in the high speed real-time visualization software system, its function is rich, simple operation completely suitable for two phase flow detection visual measurements. In 2012, Yang Chengyi and others based on double cross section ERT technology to design a set of two phase flow detection system of the bubbling bed gas holdup distribution of visualization research. Liu Yonghong et al, in 2014 ,for the solid liquid two phase flow in micro-channel test experiments, using ERT system using the Unscented kalman filter algorithm for image reconstruction with each measurement sections of two phase flow distribution of each measure the conductivity distribution in the cross section by image analysis, the fluid flow in the micro-channel[10].

\section{Clinical Medicine Application.}

Medical Electrical Impedance Tomography technology is the use of Electrical characteristics of biological tissues and organs to extract the noninvasive detection technology of human physiological information. It is used in surface of the electrode to detect human input current, and then measure the appropriate parts of the voltage variation, and the electrical impedance changes related to tissue or organ, for physiological and pathological information. This method of imaging and computed tomography (CT), nuclear magnetic resonance (NMR) is the obvious difference. EIT is functional imaging, and harmless to the human body in the process of measurement, safety, low cost, simple operation and can be repeated use for a long time, so has extensive application prospect [11].

Henderson and Webster got the first electrical impedance image in 1978, it clearly shows the location of the lungs [12]. In 1983, at the university of Sheffield B.H.Brown and D.C. Baber reported that they build EIT system in the world, studied the cardiopulmonary function imaging, imaging speed of 25 frames per second. In 1995, Robert designed a set of real-time EIT system, adopted the 16 electrodes and a public current source, can into a picture like within $80 \mathrm{~ms}$, blood perfusion can be dynamic observation. such as good application prospect in medicine. On the medical application prospect is good [13]. EIT experiment at the University of UCL is committed to the impedance of the epilepsy monitoring, has made some achievements. British De Montfort university research team developed multiple frequency breast imaging test detection system has been preliminarily the human body, can identify $5 \mathrm{~mm}$ of the tumor. 
At home, EIT research institute has been actively research, exploration and achieved certain results. Developed by the fourth military medical university in EIT bedside monitoring system, is applied to the image of the brain. Huazhong university of science and technology to build real-time monitoring patients with cardiopulmonary function change technology platform, detection in patients with cardiac ejection function, diastolic function and myocardial quality change, the disease of the tissues and organs function and structure of the real-time monitoring, it is very important and rich clinical significance. Tianjin university school of electrical and automation engineering using biological impedance technology to define focal breast cancer. Some experimental results have been achieved.

Above studies are based on the two dimensional space of the image measurement positioning, in breast detection of three dimensional electrical impedance tomography imaging, the researchers to explore. Such as the UK De Montfort university of EIT mammary gland inspection system [14], using the 4 layer ring electrode configuration and end plane electrode configuration of barrel breast measuring electrode system, and using the brine as the detection electrodes dielectric, ingeniously solved the barrel fixed electrode contact with the skin problems. At home, some scholars also put forward in the mammary gland inspection system using three dimensional electrical impedance tomography imaging and T - SCAN scanning imaging method of combining the new method, can realize three dimensional electrical impedance tomography imaging of mammary gland, and in the positive test.

EIT technology application in medicine is more widely. It can be used in cardiovascular system, blood flow distribution measurement and monitoring. In the central nervous system, it can undertake guardianship of intraventricular hemorrhage. In terms of the respiratory system, EIT can be used to to test the pulmonary edema, monitoring on lung function. In terms of digestive system, it can be carried out research of gastric function, etc

So far, the development of EIT technology has come a long way. And the researchers in actively put test technology into clinical application equipment, ultimately to serve the patients. Exciting effect has been made, shows the tantalizing prospect of electrical impedance technology. We acknowledge the EIT imaging accuracy obviously can't with common CT, PET, etc. In comparison, there are still many problems in theory and practical measurement to solve. But we should realize the advantages of EIT is distinguished from other large equipment, is miniaturization, used for portable monitoring based on the use of the object, can be used for objects can consider family and personal medical auxiliary testing equipment

\section{Electrical Resistance Tomography Imaging of Concrete.}

Reinforced concrete structure in the world, accounts for the vast majority of buildings in various building structures. As the growth of the service time of rein-forced concrete buildings, old buildings increasing, the safety and reliability of the reinforced concrete structure has gradually become a universal concern in the field of civil engineering problems. With the deterioration and failure rates escalating as this infrastructure continues to age, it is critical that better nondestructive techniques are developed to allow early detection of the deterioration process so that proper repair strategies can be applied.. Techniques that provide information on the location of the rein-forcing bars, the degree of cracking in the reinforced concrete, the distribution of chlorides in the matrix, the humidity gradients in concrete, the state of corrosion, and the depth of cover, are needed. Electrical methods, which vary in terms of costs and ease of use, are well suited for inspection and evaluation of large concrete structures. A number of studies during the last two decades have indicated that electrical measurements carry information on several properties of concrete. The Figure 2, 3 and 4 are the electrical resistance tomography for the reconstruction of clutter in the concrete, see [15]. 


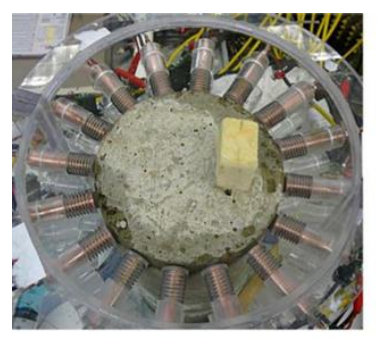

a

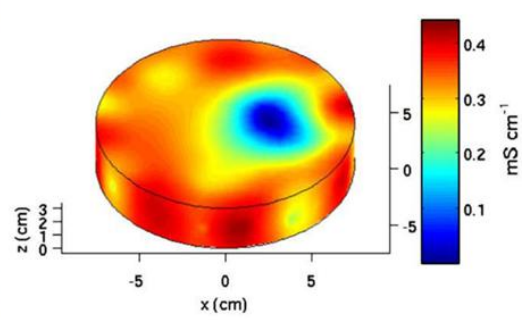

b

Fig.2. a) A specimen including a resistive polyurethane block. b) The ERT reconstruction[15].

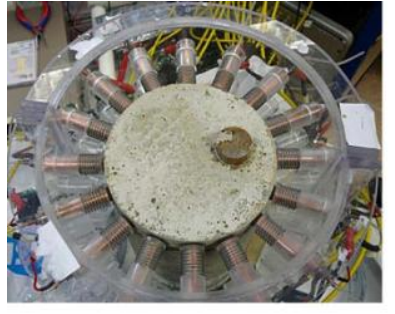

a

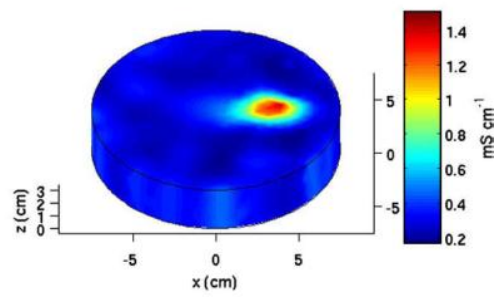

b

Fig.3. A steel rod cast in concrete. a) The specimen. b) The reconstructed conductivity distribution[15].

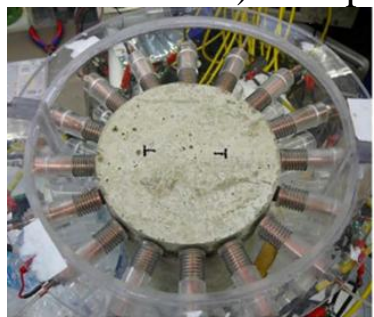

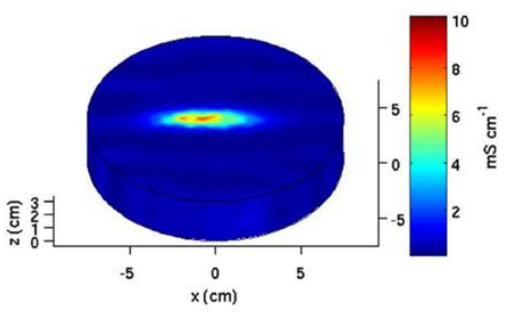

Fig.4. a) A specimen including a horizontal steel bar. Direction of the bar is from left to right. The ends of the bar are indicated in the photo with black marks. b) The reconstructed conductivity distribution[15].

In 2003, j. f. Lataste using electrical resistance tomography technology and the success of the implementation for concrete detection of cracks[16]. In 2004, J.Zhang and others using ERT system for the rebar corrosion rate measurement [17]. In 2006, n. Ozyurt using electrical resistance tomography technology that was applied to the distribution of fiber material in concrete detection. J.M. Loche using ERT technology to the humidity of concrete structure was tested. In 2010, the ERT technology of concrete structure is used for three dimensional imaging [15]. It demonstrates the applicability of using ERT for evaluating the properties of concrete. Concrete specimens cast with a polyurethane block, horizontal steel bar, a vertical steel bar, and plastic plates were measured using ERT. The results indicate that different types of inclusions can be detected and localized using this method. Potential applications of ERT in the construction industry include detection of the thickness of the concrete cover on top of reinforcement bar and estimation of crack depths and moisture distributions. In addition, the information of the reinforcement bars location provided by ERT could be utilized in diagnosing the corrosion rate of reinforcement bars. In 2015, Milad Hallaji and others used ERT technology to achieve the dynamic imaging measurement for the permeability to water in the concrete [18].

\section{Conclusions}

Above, we can see that the ERT has the unique superiority and broad development. Along with the advance of science and technology, ERT technology has developed rapidly, expanding its application field. Of course, we also demand a lot of basic research. Such as to study the suitable acquisition system, realize the optimization of the imaging algorithm, the research has the memory function of imaging software and hardware equipment, formulate the corresponding testing standards. The current study is still more concentrated in the laboratory application research, the scene of the need to implement from the lab to the real industrial applications. We firmly believe that electrical resistance tomography technology with attractive potential and wide application prospect. 


\section{References}

[1]Dyakowski T. 1996. Process tomography applied to multi-phase flow measurement, Meas. Sci. Technol 7(3):343-353.

[2]Li Haiqing, Qiao Hetang. 1996. Multiphase flow detection technology development. Petroleum Industry Press:57-56.

[3]Jackson J D. 2004. Classical electrodynamics, John Wiley and Sons, China High Education Press.

[4]Yang W Q, et al. 1999. An image-reconstruction algorithm based on landweber's iteration method for electrical capacitance tomography, Meas. Sci. Technol10(11):1065-1069.

[5]Dickin F J, Wang M. 1996. Electrical resistance tomography for process applications, Meas. Sci. Techno 7(3):247-260.

[6]Claude C -B.1997. Regularized Reconstruction in Electrical impedance tomography using a variance uniformization constraint, IEEE T. Med. Imaging 16(5):562-571.

[7]Primrose, K, \& Qiu, C. 1999. Performance and application studies of an electrical resistance tomography system. Ref.

[8]Y Dai, M Wang. 2005. 3-D visualization of a swirling flow using electrical resistance tomography, World Congress on Industrial Process Tomography 362-367.

[9]Deng, X. 2001. The design of a dual plane art system for cross correlation measurement of bubbly gas/liquid pipe flow. Measurement Science \& Technology, volume 12, 8, 1024-1031.

[10]Wang Pai. 2012. ERT technology and its application in two phase flow detection. Xidian University.

[11]Zhang Lifeng. 2010. Research on exciting measuring modes and image reconstruction algorithms for electrical tomography. Tianjin University..

[12]H.Griffiths. 1990. Electrical Impedance Tomography. Physical Medical6(3-4)109-116.

[13]Robert W, et al. 1995. A Real-time Electrical Impedance Tomography System for Clinical Use-design and Preliminary Results. IEEE BEM.42(2)133-140.

[14]Zadehkoochak M, et al.1990. A transporter implemented algorithm for electrical impedance tomography. Clinic Physiology Meas11(3):223-230.

[15]Karhunen, K, et al. 2010. Electrical resistance tomography imaging of concrete. Cement \& Concrete Research40(1): 137-145.

[16]J.F. Lataste, et al. 2003. Electrical resistivity measurement applied to cracking assessment on reinforced concrete structures in civil engineering, NDT \& E International 36 383-394.

[17]J. Zhang, et al. 2004. Surface measurement of corrosion of reinforcing bar in concrete -3: effect of geometry and material properties, ACI Materials Journal 101 273-280.

[18]Hallaji, M. Seppänen, A. 2015. Electrical resistance tomography to monitor unsaturated moisture flow in cementations materials. Cement \& Concrete Research10-18. 